\title{
NÚMERO DE LARVAS DE Oreochromis sp, EN DOS PROPORCIONES REPRODUCTIVAS CON DOS SISTEMAS DE MANEJO
}

\author{
Heriberto Andrés Giraldo Lizarazo', Hermes Rafael Pineda Santis ${ }^{2}$ \\ ${ }^{1}$ Ingeniero Agropecuario. Profesional Independiente. andres-93giraldo@hotmail.com \\ ${ }^{2}$ Biólogo, M.Sc en Biología, Grupo de Investigación Acuícola, Profesor Asociado, Facultad de Ciencias \\ Agrarias, Politécnico Colombiano Jaime Isaza Cadavid, Carrera 48 No. 7-151 Av. Las Vegas, Medellín, \\ Colombia.hrpineda@elpoli.edu.co
}

\begin{abstract}
RESUMEN
Se planteó como objetivo general evaluar el número de larvas de Oreochromis sp en proporciones reproductivas 3:1 y 3:2 (relación Hembras:Machos) y los parámetros fisicoquímicos del agua en dos sistemas de manejo (recambio de agua y aireación artificial), realizado en el Centro Experimental Piscícola en San Jerónimo (Antioquia). Los datos fueron procesados mediante el paquete estadísticos PAST $^{\odot}$ para estadística descriptiva y análisis de comparación. Los resultados mostraron que, los estanques con aireación artificial tuvieron un mayor número final (58.630 larvas), respecto al recambio de agua (24.451 larvas), sumando ambas proporciones. Los parámetros fisicoquímicos del agua presentaron diferencias significativas $(p<0.05)$ entre ambos manejos, estando dentro de los rangos óptimos para el hibrido, con niveles de Oxígeno entre 7.0-10.7 $\mathrm{mg} / \mathrm{L}$, temperaturas entre $25.7-29.8^{\circ} \mathrm{C}$ y $\mathrm{pH}$ entre $8.5-9.0$, considerando ambas proporciones. El manejo con aireación presentó el mayor número de larvas en las dos proporciones.
\end{abstract}

Palabras Clave: Aireación, Recambio de agua, Reproducción, Tilapia

Recibido: 26 de abril de 2019. Aceptado: 27 de Noviembre de 2019.

Received: April 26, 2019. $\quad$ Accepted: November 26, 2019.

\section{NUMBER OF LARVAE OF Oreochromis sp IN TWO REPRODUCTIVE PROPORTIONS WITH TWO MANAGEMENT SYSTEMS}

\begin{abstract}
The general objective was to evaluate the number of Oreochromis sp larvae considering the reproductive proportions 3:1 and 3:2 (Female:Males ratio) and the physicochemical parameters of water in two management systems (water exchange and artificial aeration), at the Centro Experimental Piscícola in San Jerónimo (Antioquia). The data were processed using the statistical package $P A S T^{\odot}$ for descriptive statistics and comparison analysis. The results showed that, artificially aerated ponds had a higher final number (58.630 larvae), compared to the water exchange (24.451 larvae), adding both proportions. The physicochemical parameters of the water presented significant differences $(p<0.05)$ between both managements, but they were within the optimum ranges for the hybrid, with oxygen levels between 7.0-10.7 mg/L, temperatures between 25.7-29.8 ${ }^{\circ} \mathrm{C}$ and $\mathrm{pH}$ between 8.5-9.0, considering both proportions. The management with aeration presented the highest number of larvae in both proportions.
\end{abstract}

Keywords: aeration, water exchange, reproduction, Tilapia

Cómo citar este artículo: H. Giraldo, H. Pineda. "Número de larvas de oreochromis sp, en dos proporciones reproductivas con dos sistemas de manejo", Revista Politécnica, vol. 15, no.30 pp.25-31, 2019. DOI: 10.33571/rpolitec.v15n30a3 


\section{INTRODUCCIÓN}

La tilapia, específicamente el género Oreochromis sp, es el segundo grupo de peces más productivos en el mundo con 4200 millones de ton/año [1], que la hacen muy apetecida en muchas regiones subtropicales y tropicales, por sus características tales como: filete suave y de buen sabor, alta rusticidad que los hace manipulables en cautiverio, soporta amplios rangos de calidad de agua, muy resistentes a las enfermedades, fácil reproducción y con hábitos omnívoros con tendencia a herbívoros [2], [3], [4].

Las variaciones ambientales sobre los reproductores en una unidad productiva, en este caso, el manejo de la calidad del agua y la disponibilidad de reproductores para una mejor eficacia reproductiva, son de vital importancia para establecer las condiciones apropiadas en el cultivo, ya que de ellos depende la venta de semilla, un mercado de alta demanda, o para el uso en el mismo sistema productivo con el fin de comercializar la carne [5].

El calentamiento global y la contaminación antrópica, han disminuido el volumen de agua disponible para las producciones piscícolas, que manejan el ingreso de agua de forma directa al sistema, a través del recambio de agua, por lo que se deben generar alternativas de manejo capaces de dar solución a esta problemática [6]. Los mecanismos de aireación artificial, encargados de incorporar el Oxígeno atmosférico al agua, ayudan a plantear una estrategia de manejo para conocer su efecto sobre la producción de larvas. Asimismo, la optimización de los reproductores con mayores posibilidades de apareamiento, redundaría en un mayor beneficio productivo.
Por ende, se plantea como objetivo general, evaluar el número de larvas de tilapia roja Oreochromis sp, considerando las proporciones reproductivas 3:1 y 3:2 (relación Hembras:Machos), en un sistema con aireación artificial y otro con recambio de agua, para establecer sus diferencias y tomar decisiones técnicas.

\section{MATERIALES Y MÉTODO}

El ensayo se realizó en el Centro Experimental Piscícola (CEPI), Vereda Loma Hermosa, en el municipio de San Jerónimo (Antioquia) (coordenadas $6^{\circ} 26^{\prime} 49.88^{\prime \prime} \mathrm{N}$ y $75^{\circ} 43^{\prime} 55.42^{\prime \prime}$ W), a $780 \mathrm{msnm}$, temperatura media de $28^{\circ} \mathrm{C}$, humedad relativa del $50 \%$ y zona de vida: Bosque seco tropical (bs-T) [7]. El agua se obtuvo de la quebrada Guaracú, perteneciente a la cuenca del río Cauca, con los siguientes parámetros fisicoquímicos: temperatura: $23-25{ }^{\circ} \mathrm{C}, \mathrm{pH}: 7.5-9.0$, dureza 120 ppm, Oxígeno $7.8 \mathrm{mg} / \mathrm{L}$, amonio 1.46 ppm, cloro 6 ppm, topografía pendiente, 900 $\mathrm{mm}$ de precipitación anual y alta turbidez en épocas de invierno.

Se realizó el sexado previo a los animales destinados a la reproducción para identificar y separar hembras y machos con características fenotípicas de color homogéneo y talla reproductiva ajustada a la madurez sexual. Se utilizaron 120 reproductores (90 hembras y 30 machos), manteniendo la proporción 3:1, y 150 reproductores (90 hembras y 60 machos), para una proporción 3:2. Los animales se pesaron en una balanza analítica $\left(\mathrm{OHAUS}{ }^{\circledR}\right.$, EEUU), y se realizó un tratamiento con sal marina para disminuir el estrés causado por la manipulación. Los pesos promedios en hembras y machos se observan en la Tabla 1.

Tabla 1. Peso promedio general de hembras y machos de tilapia roja Oreochromis $s p$ en dos proporciones reproductivas.

\begin{tabular}{lcc|cc}
\hline & \multicolumn{2}{c|}{ Proporción 3:1 } & \multicolumn{2}{c}{ Proporción 3:2 } \\
& \multicolumn{2}{c}{$(15: 5$ por estanque) } & \multicolumn{2}{c}{$(15: 10$ por estanque $)$} \\
\hline & Hembras $(\mathrm{g})$ & Machos $(\mathrm{g})$ & Hembras $(\mathrm{g})$ & Machos $(\mathrm{g})$ \\
Recambio & $371 \pm 56$ & $567 \pm 57$ & $371 \pm 56$ & $483 \pm 28$ \\
Aireación & $324 \pm 16$ & $587 \pm 49$ & $324 \pm 16$ & $493 \pm 25$ \\
Total & 90 & 30 & 90 & 60 \\
\hline
\end{tabular}


Se seleccionaron 12 estanques en tierra, recubiertos con una capa fina de cemento, no mayor a $2 \mathrm{~cm}$ de grosor, los cuales se vaciaron, lavaron, desinfectaron y llenaron con un volumen promedio efectivo de agua de $7.7 \mathrm{~m}^{3}$, sugiriendo que se pueden introducir entre 20 y 25 animales/estanque.

Seis de los estanques fueron utilizados para el recambio de agua, ajustando su ingreso a 6 $\% /$ hora. Los otros seis se acondicionaron con dos parrillas por estanque, con un metro de manguera difusora Aero Tube ${ }^{\mathrm{TM}}$, que transfirió $0.061 \mathrm{~kg} \mathrm{O} /$ hora/metro, cada una en el fondo, que recibió aire atmosférico a presión desde un aireador de $1 / 2 \mathrm{HP}$. Estos estanques no tuvieron recambio de agua, por lo que el nivel de Oxigeno fue solamente por medio de la manguera difusora.

De los seis estanques en cada manejo, tres tuvieron una proporción 3:1 (Hembras:Machos) y los otros tres, una proporción 3:2 (Hembras:Machos), con lo cual

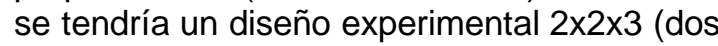
proporciones, dos manejos y tres repeticiones). Los reproductores se alimentaron con concentrado de $30 \%$ de proteína, con una ración de $0,8 \%$ de su biomasa, en una frecuencia de dos veces al día (10:00 AM y 3:00 PM).

A partir del momento que se observaron las larvas en los estanques de reproducción, aproximadamente entre 15 a 20 días, se procedió a la recolección en la mañana, cada dos o tres días. No se permitió más tiempo porque implica problemas con la eficiencia en el proceso de reversión de las larvas y pérdidas por canibalismo. El conteo se realizó de forma masal, mediante extrapolación del peso obtenido dividido por 0,022 g (peso promedio de una larva), para obtener el número total recogido. La recolección se realizó antes de la alimentación con sistemas de redes muy finas para evitar el maltrato de las crías y su mortalidad. El tiempo de recolección fue de cuatro semanas.

Los reproductores tuvieron un manejo en campo, sujeto a las Buenas Prácticas Acuícolas. De esta manera, se consideraron las normas ambientales vigentes en la granja, relacionados con el uso de anestésicos y manejo general para un menor estrés de los animales, aspectos que fueron avalados como riesgo mínimo, según radicado 201701009661, por el Comité de Ética (CEPI) de la Dirección de Investigación y Posgrado.

Se tomaron las siguientes variables fisicoquímicas del agua, dos veces por semana (martes y viernes): nivel de Oxígeno $(\mathrm{mg} / \mathrm{L})$ con el oxímetro PINPOINT $\|^{\circledR}$ (American Marine Inc - USA), temperatura $\left({ }^{\circ} \mathrm{C}\right)$ y $\mathrm{pH}$ observados con el medidor multiparámetro (Oakton ${ }^{\circledR}$ México), el color por observación directa y la turbidez del agua con el disco de Secchi.

Los datos fueron registrados en el aplicativo Excel Microsoft, y procesados en el paquete estadístico $\mathrm{PAST}^{\circledR}$, luego de verificar el cumplimiento de supuesto de normalidad mediante la prueba Shapiro-Wilk. A partir de los datos se obtuvo la estadística descriptiva y el análisis de varianza, entre las variables de fisicoquímicas del agua para las comparaciones de medias (Tukey), en las proporciones y manejos considerados.

\section{RESULTADOS Y DISCUSIÓN}

\subsection{Parámetros fisicoquímicos del agua}

Los parámetros fisicoquímicos del agua mostraron diferencias significativas $(p<0.05)$ entre los sistemas de manejo, redundando en un mayor número de larvas en el sistema de aireación artificial. Aún con los cambios observados, los rangos obtenidos estuvieron dentro de las condiciones óptimas del cultivo para el hibrido [8], [9].

El nivel de Oxígeno mostró diferencias significativas $(p<0.05)$ entre el recambio de agua y la aireación en ambas proporciones, observándose un mayor valor en los

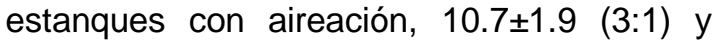
8.0 \pm 1.7 (3:2) $\mathrm{mg} / \mathrm{L})$, comparado con los estanques de recambio de agua $8.9 \pm 1.4$ (3:1) y $7.0 \pm 1.4(3: 2) \mathrm{mg} / \mathrm{L}$ ) (Tabla 2). El nivel de Oxígeno siempre fue más alto en horas del mediodía e inicio de la tarde, debido a que la luminosidad fue más alta, favoreciendo la fotosíntesis de los organismos fitoplanctónicos en los estanques, lo cual concuerda con otros autores [10], [11]. Los valores registrados de Oxígeno son superiores a $5 \mathrm{mg} / \mathrm{L}$, lo cual está dentro del rango de cultivo del hibrido [8], [9]. 
La renovación constante del agua (6\%/hora), permitió la remoción de sedimentos, incorporación de Oxígeno y rompimiento del ciclo biológico dentro del estanque, disminuyendo la eutroficación, debido a la renovación total de su volumen a las 17 horas. Lo contrario sucedió en los estanques de aireación, donde hubo mayor sedimentación y presencia de organismos fotosintéticos aumentando la eutroficación [10], acentuando el color verde.

El manejo con aireación artificial, presentó condiciones más apropiados a la exigencia de los animales, por este motivo hubo un mayor número de larvas. Con ello, se suplieron los requerimientos de Oxígeno, y se observó una mayor estabilidad en su oxigenación durante el día, comparados a los estanques de recambio, donde presentaron mayor variación, debido a los fenómenos ambientales entre el día y la noche.

Una disminución de la cantidad de Oxígeno disuelto en el agua, está afectada por los procesos de descomposición de la materia orgánica, alimento no consumido, heces, animales muertos, aumento de la tasa metabólica por el incremento en la temperatura, respiración de fitoplancton y zooplancton, salida del oxígeno del agua hacia la atmósfera, días opacos que no permiten a las algas la producción de suficiente oxígeno, aumento de sólidos en suspensión, residuos de sedimentos en el agua $y$ altas densidades de siembra, ocasionando inapetencia y muerte de los reproductores, enfermedades en branquias, inmunosupresión y disminución de la capacidad reproductiva [12], [13].

Respecto a la temperatura, la incorporación continua de agua fresca a los estanques de recambio, produjo una reducción de la temperatura, alcanzando valores menores que en los estanques con aireación artificial $(p<0.05)$, donde no hubo incorporación desde la fuente natural (Tabla 2). El agua retenida en los estanques con aireación artificial, presentó un aumento significativo en $3^{\circ} \mathrm{C}$, favoreciendo el comportamiento reproductivo en los peces, mediante el acercamiento prenupcial por efecto de la atracción hormonal [14] y una mayor eutroficación para una alimentación alternativa, por el consumo de la productividad primaria, debido al hábito herbívoro del híbrido [15].

La temperatura promedio del agua, en los estanques con aireación artificial, presentó condiciones más favorables para la tilapia roja Oreochromis sp, comparada con lo observado en los estanques con recambio, donde fue mucho menor. Este aspecto es importante, ya que la temperatura corporal depende exclusivamente del medio acuático donde se encuentre. El rango óptimo para el cultivo del hibrido fluctúa entre $25-30{ }^{\circ} \mathrm{C}$ [8], [9].

Los valores de $\mathrm{pH}$ en ambos manejos tuvieron diferencias significativas $(p<0.05)$, pero no en las dos proporciones reproductivas $(p>0.05)$ (Tabla 2). Es de resaltar que los valores obtenidos (Recambio 8.5 y Aireación 9.0), estuvieron dentro del rango óptimo para el hibrido [8], [9], con una ligera tendencia a la alcalinidad, debido a mayores concentraciones de carbonato y bicarbonato, que mantuvieron el pH más estable. En una exagerada productividad primaria (mayor eutroficación), el pH se elevaría desencadenando una precipitación de ellos, que se vería como un polvo blanco sobre el fondo [13], lo cual no sucedió en esta observación.

Valores por encima o por debajo de $\mathrm{pH}$, fuera del rango de tolerancia, causan problemas reproductivos y generan cambios de comportamiento en los peces como letargia, inapetencia y retardan el crecimiento en los animales [4].

Durante la recolección de larvas, no se presentó turbidez por material mineral particulado o lodos. Hubo periodos de material orgánico (eutroficación), específicamente en los estanques con aireación debido al no recambio y estabilidad de las condiciones ambientales, que hizo propicio el mayor crecimiento de la productividad primaria [16].

Tabla 2. Parámetros fisicoquímicos del agua en dos proporciones reproductivas y dos sistemas de manejo en reproductores de tilapia roja Oreochromis $s p$. 


\begin{tabular}{lccc|ccc}
\hline & $\begin{array}{c}\text { Nivel de } \\
\text { Oxígeno } \\
(\mathrm{mg} / \mathrm{L})\end{array}$ & $\begin{array}{c}\text { Temperatura } \\
\left({ }^{\circ} \mathrm{C}\right)\end{array}$ & $\mathrm{pH}$ & $\begin{array}{c}\text { Nivel de } \\
\text { Oxígeno } \\
(\mathrm{mg} / \mathrm{L})\end{array}$ & $\begin{array}{c}\text { Temperatura } \\
\left({ }^{\circ} \mathrm{C}\right)\end{array}$ & $\mathrm{pH}$ \\
\hline Recambio & $8.9 \pm 1.4^{\mathrm{a}}$ & $25.7 \pm 1.9^{\mathrm{a}}$ & $8.5 \pm 0.1^{\mathrm{a}}$ & $7.0 \pm 1.4^{\mathrm{a}}$ & $26.5 \pm 2.1^{\mathrm{a}}$ & $8.5 \pm 0.1^{\mathrm{a}}$ \\
Aireación & $10.7 \pm 1.9^{\mathrm{b}}$ & $28.7 \pm 2.8^{\mathrm{b}}$ & $9.0 \pm 0.1^{\mathrm{b}}$ & $8.0 \pm 1.7^{\mathrm{b}}$ & $29.8 \pm 3.0^{\mathrm{b}}$ & $9.0 \pm 0.1^{\mathrm{b}}$ \\
\hline
\end{tabular}

\subsection{Parámetros de producción de larvas}

Las primeras larvas observadas fueron a los 12 días en los estanques con aireación artificial, confirmando que, las condiciones del agua con este manejo fue el más adecuado.

La mayor cantidad de larvas recolectadas en los estanques con aireación artificial, esto es, 30.091 (3:1) y 28.539 (3:2), mostraron que este ambiente fue el más apropiado, permitiendo un mejor entorno para la reproducción $y$, por ende, la producción de larvas con algo más de la mitad (56\%), comparado con el sistema de manejo con recambio, es decir, 12.846 (3:1) y 11.605 (3:2) (Figura 1).

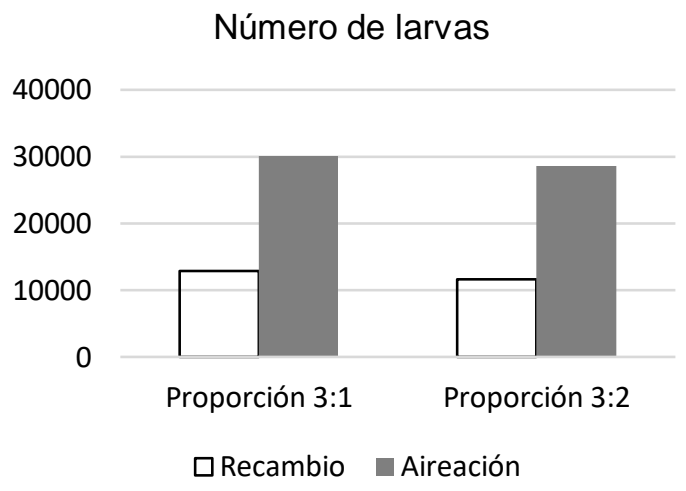

Figura 1. Número de larvas recolectadas en dos manejos y dos proporciones reproductivas en tilapia roja Oreochromis $s p$.

Espejo \& Torres (2001) [11] sugirieron que, la cantidad de larvas viables está en una relación de 1.8 larvas por gramo de peso de las hembras, por lo que esta relación se ajusta a lo observado en el manejo con aireación artificial, es decir, tomando $371 \mathrm{~g}$, peso promedio general de las hembras, multiplicado por 1.8, por 15 hembras por estanque, por tres repeticiones, daría una cifra de 30.051, aproximado a lo obtenido. Por el contrario, para el manejo con recambio no se ajustó a lo recolectado en los estanques (26.244 larvas), evidenciando que la cantidad depende de las condiciones ambientales, esto es, de manejo en el estanque.
Los valores parciales de larvas obtenidas por estanque, en cada manejo y proporción reproductiva, se observan en la Tabla 3.

Tabla 3. Número de larvas obtenidas por estanque considerando el manejo y la proporción reproductiva

\begin{tabular}{ccccccc}
\hline & \multicolumn{3}{c}{ Recambio } & \multicolumn{3}{c}{ Aireación } \\
\hline & $\mathrm{R} 1$ & $\mathrm{R} 2$ & $\mathrm{R} 3$ & $\mathrm{~A} 1$ & $\mathrm{~A} 2$ & $\mathrm{~A} 3$ \\
\hline $3: 1$ & 3348 & 5262 & 4236 & 12502 & 10012 & 7577 \\
$3: 2$ & 2998 & 4208 & 4399 & 9362 & 7906 & 11271 \\
\hline
\end{tabular}

La luminosidad favoreció la productividad primaria en los estanques con aireación, ofreciendo un alimento extra, creando un mayor estimulo reproductivo en los animales, y una mayor sobrevivencia de larvas. Las partículas orgánicas tales como los detritus de plantas y animales de varios tamaños, también estarían suspendidas en el agua junto con las bacterias, las algas, restos de alimento, materia fecal y varios tipos de fitoplancton y zooplancton [17]. Por consiguiente, la productividad natural presente en estos estanques, permitió la formación de redes tróficas que, a diferencia de los estanques de recambio, hubo renovación constante de agua, sin alcanzar un punto de eutroficación avanzado. Se observó que los reproductores tuvieron un color rosado más acentuado y brillante.

Meyer (2004) [10] sugiere que, despues de periodos largos de apareamiento, se debe dar un descanso a los reproductores con los que se esta trabajando, la recomendación es ingresar animales nuevos al sistema, con esto se evita el desgaste de los reproductores y su vida reproductiva se prolonga mas tiempo.

La proporción sugerida por varios autores en cultivos de tilapia, es superar el número de hembras con relación a los machos (Tabla 4), por lo que sigue siendo válida la observación.

Tabla 4. Proporción Hembras Machos en reproducción de tilapias. 


\begin{tabular}{|c|c|}
\hline Hembras: Machos & Referencia \\
\hline $2: 1$ & Alcántar et al, 2014 [9] \\
\hline 3:1 & $\begin{array}{l}\text { Hussain, } 2004 \text { [2] } \\
\text { Merino et al, } 2006 \text { [18] }\end{array}$ \\
\hline $3: 1 \circ 2$ & $\begin{array}{l}\text { Cantor, } 2007 \text { [8] } \\
\text { Nicovita, } 2007 \text { [12] }\end{array}$ \\
\hline 2 o $3: 1$ & $\begin{array}{l}\text { FAO, } 2009 \text { [19] } \\
\text { Salama, } 1996 \text { [20] }\end{array}$ \\
\hline $3 \circ 5: 1$ & $\begin{array}{l}\text { Hsien-Tsang \& } \\
\text { Quintanilla, 2008 [21] }\end{array}$ \\
\hline
\end{tabular}

La proporción 3:1 ofreció el mayor número de larvas que la 3:2, sugiriendo que la menor densidad de los reproductores proporcionó el mejor manejo. La recolección de larvas evidenció la importancia del mayor número de las hembras respecto al de los machos.

Las densidades consideradas en este ensayo fueron de 1.2 (3:1) y 1.5 (3:2) animales $/ \mathrm{m}^{2}$, coincidiendo con un sistema de cultivo extensivo (0.5-3 animales $\left./ \mathrm{m}^{2}\right)$ [12]. Además, Cantor (2007) [8], sugirió que los reproductores no deben superar $1 \mathrm{~kg} / \mathrm{m}^{2}$, debido a que se disminuye la postura. En esta observación el peso de los reproductores osciló entre 0.472 y $0.630 \mathrm{~kg} / \mathrm{m}^{2}$, considerando tanto el manejo y la proporción.

La nutrición es uno de los principales factores asociados a la calidad de los gametos en los reproductores. Por ello, la productividad primaria, en el caso del manejo con aireación, se convirtió en un estímulo de alimentación heterotrófica, mediante el suministro de materia orgánica y detritus al ecosistema, que redundó en la frecuencia de desove, número de ovas por desove y la calidad de ovas y larvas producidas [22], [9]. En general, los alimentos con 25-35\% de proteína cruda, proveen los nutrientes necesarios para los reproductores, alimento similar al utilizado en la etapa de engorde [23], [12].

\section{CONCLUSIONES}

Los estanques con aireación artificial, obtuvieron un mayor número de larvas (58.630), comparados con los obtenidos en los estanques con recambio de agua (24.451). La proporción más efectiva fue $3: 1$, considerando el ambiente más apropiado, la menor densidad y la mayor disponibilidad de productividad primaria, en los estanques con aireación artificial. Los parámetros fisicoquímicos del agua $(\mathrm{pH}$, temperatura, nivel de oxígeno y turbidez), estuvieron dentro del rango óptimo para los reproductores de tilapia roja Oreochromis sp, en ambos manejos.

\section{AGRADECIMIENTOS}

Agradecimientos al personal técnico y administrativo del Centro Experimental Piscícola (CEPI) en San Jerónimo (Antioquia). A la Dirección de Investigación y Posgrado, por el apoyo financiero través de la Convocatoria Interna para estudiantes 2017 (Radicado: 201701009590).

\section{REFERENCIAS}

[1] FAO (Organización de las Naciones Unidas para la Alimentación y la Agricultura). El estado mundial de la pesca y la acuicultura: cumplir con los objetivos de desarrollo sostenible. Roma. Licencia: CC BY-NC-SA 3.0 IGO. 2018.

[2] Hussain, M.G. Farming of tilapia: breeding plans, mass seed production and aquaculture techniques. Momin Offset Press, Djaka (Bangladesh). 2004.

[3] Zimmermann, S. Reproducción de tilapias. En: Reproducción de peces en el trópico. (Eds P.V. Daza, M.A. Landines Parra, A. Sanabria Ochoa), Ministerio de Agricultura y Desarrollo Rural, Instituto Colombiano de Desarrollo Rural (INCODER), Subgerencia de Pesca y Acuicultura, Universidad Nacional de Colombia, Facultad de Medicina Veterinaria y de Zootecnia. Bogotá DC. 2005.

[4] Saavedra Martínez, M.A. Manejo del cultivo de tilapia. USAID, Coastal Resorurces Center, University of Hawaii HILO, Departamento de Tecnología y Arquitectura. Facultad de Ciencia, Tecnología y Ambiente. Universidad Centroamericana. Managua (Nicaragua). 2006.

[5] Senhorini, J.A., Landinez-Parra, M.A. Generalidades sobre manejo y selección de reproductores de peces reofilicos. En: Reproducción de peces en el trópico. (Eds P.V. Daza, M.A. Landines Parra, A. Sanabria Ochoa), Ministerio de Agricultura y Desarrollo Rural, 
Instituto Colombiano de Desarrollo Rural (INCODER), Subgerencia de Pesca y Acuicultura, Universidad Nacional de Colombia, Facultad de Medicina Veterinaria y de Zootecnia. Bogotá DC. 2005.

[6] De Silva, S.S., Soto, D. El cambio climático y la acuicultura: repercusiones potenciales, adaptación y mitigación. En: Consecuencias del cambio climático para la pesca y la acuicultura: visión de conjunto del estado actul de los conocimientos cientificos. Documento técnico de pesca y acuicultura 530 (FAO). (Eds K. Cochrane, C. De Young, D. Soto, T Bahri), Roma. 2012.

[7] Holdridge, L. Ecología Basada en Zonas de Vida. Editorial IICA. Costa Rica. 1979.

[8] Cantor, F. Manual de producción de tilapia. Secretaria de Desarrollo Rural del Estado de Puebla. México. 2007.

[9] Alcántar-Vázquez, J.P., Santos-Santos, C., Moreno-de la Torre, R. y Antonio-Estrada, C. Manual para la producción de supermachos de tilapia del Nilo (Oreochromis niloticus). UNPAPIFI, Oaxaca. México. 2014.

[10] Meyer, D. Introducción a la Acuacultura. Escuela Agrícola Panamericana. Zamorano (Honduras). 2004.

[11] Espejo, C., Torres, E. Cultivo de las tilapias roja Oreochromis sp y plateada Oreochromis niloticus. En: Fundamentos de acuicultura continental. (Eds H. Rodríguez, P.V. Daza, M. Carrillo), Ministerio de Agricultura y Desarrollo Rural. Instituto Nacional de Acuicultura y Pesca (INPA). Bogotá DC. 2001.

[12] Nicovita. Manual de crianza de la tilapia. Alicorp. Lima (Perú). 48 p. 2007.

[13] Argumedo, E., Rojas, H. Manual de piscicultura con especies nativas. Asociación de Acuicultores del Caqueta (ACUICA). Plan Nacional de Desarrollo Alternativo. República de Colombia.Produmedios. Bogotá. 2008.

[14] Huertas, M., Almeida, O.G., Canario, A. y Hubbard, P. Tilapia male urinary pheromone stimulates females reproductive axis. Gen. Comp. Endoc., 196, 106-111, 2014.
[15] Mjoun, K., Rosentrater K. y Brown, M.L. Tilapia: Environmental Biology and Nutritional Requirements. Fact Sheets Paper. 164, 2010.

[16] Rodríguez, H., Anzola, E. La calidad del agua y la productividad de un estanque en la acuicultura. En: Fundamentos de acuicultura continental. (Eds H. Rodríguez, P.V. Daza, M Carrillo), Ministerio de Agricultura y Desarrollo Rural. Instituto Nacional de Acuicultura y Pesca (INPA). Bogotá DC. 2001.

[17] Stickney, R. Understanding and maintaining water quality. En. Aquaculture: an introductory text. Texas A \& M University. CABI Publishing. Cambridge. USA. 2005.

[18] Merino, M.C., Salazar, G. y Gómez, D. Guía práctica de piscicultura en Colombia: una valiosa herramienta para el usuario. Ministerio de Agricultura y Desarrollo Rural. Instituto Colombiano de Desarrollo Rural (INCODER). Subgerencia de Pesca y Acuicultura, Bogotá DC, 2006.

[19] FAO (Organización de las Naciones Unidas para la Alimentación y la Agricultura). Oreochromis niloticus. En: Cultured aquatic species fact sheets. (Eds J.E. Rakocy, V. Crespi, M. New. CD-ROM (multilingual). 2009.

[20] Salama, M.E. Effects of sex ratio and feed quality on mass production of Nile tilapia Oreochromis niloticus (L.), Alevines. Aqua Res., 27, 581-90, 1996.

[21] Hsien-Tsang, S., Quintanilla, M. Manual sobre reproducción y cultivo de tilapia. Centro de Desarrollo de la Pesca y la Acuicultura (CENDEPESCA). Ministerio de Agricultura y Ganadería (MAG) y el Ministerio de Medio Ambiente y Recursos Naturales (MARN). Conceptos Ed, El Salvador, 2008.

[22] Rodríguez, H. El ecosistema de un estanque y la importancia del manejo para la nutrición y alimentación en acuicultura. En: Fundamentos de nutrición y alimentación en acuicultura. (Eds M.P. Soler, H. Rodríguez, P.V. Daza), Ministerio de Agricultura y Desarrollo Rural. Instituto Nacional de Acuicultura y Pesca (INPA). Bogotá DC. 1996.

[23] Baltazar, P.M. La tilapia en el Perú: acuicultura, mercado y perspectiva. Rev. Per. Biol., 13, 267-273, 2007. 